LBL-36271

UC-406

\title{
Scintillator Characterization Using the LBL Pulsed X-ray Facility
}

\author{
W.W. Moses, S.E. Derenzo, M.J. Weber, S.C. Blankespoor, M.H. Ho, and A.C. West \\ Life Sciences Division \\ Lawrence Berkeley Laboratory \\ University of California \\ Berkeley, California 94720
}

October 1994

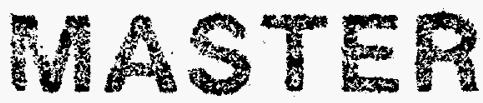

This work was supported by the U.S. Department of Energy under Contract No. DE-AC03-76SF00098, and by Public Health Service Grants P01 25840 and R01 CA48002. 


\section{DISCLAIMER}

This report was prepared as an account of work sponsored by an agency of the United States Government. Neither the United States Government nor any agency thereof, nor any of their employees, make any warranty, express or implied, or assumes any legal liability or responsibility for the accuracy, completeness, or usefulness of any information, apparatus, product, or process disclosed, or represents that its use would not infringe privately owned rights. Reference herein to any specific commercial product, process, or service by trade name, trademark, manufacturer, or otherwise does not necessarily constitute or imply its endorsement, recommendation, or favoring by the United States Government or any agency thereof. The views and opinions of authors expressed herein do not necessarily state or reflect those of the United States Government or any agency thereof. 


\section{DISCLAIMER}

Portions of this document may be illegible in electronic image products. Images are produced from the best available original document. 


\title{
Scintillator Characterization using the LBL Pulsed X-ray Facility*
}

\author{
W. W. Moses, S. E. Derenzo, M. J. Weber, S. C. Blankespoor, M. H. Ho, and A. C. West \\ Lawrence Berkeley Laboratory, University of California, Berkeley, CA 94720
}

\begin{abstract}
We have developed a bench-top pulsed x-ray system for measuring scintillation properties of compounds in crystal or powdered form. The source is a light-excited $x$-ray tube that produces $40 \mathrm{X}$-ray photons (mean energy $18.5 \mathrm{keV}$ ) per steradian in each $100 \mathrm{ps}$ fwhm pulse. The repetition rate is adjustable from 0 to $10^{7}$ pulses per second. The fluorescent emanations from the $\mathrm{x}$-ray excited samples are detected with either a sapphire-windowed microchannel plate photomultiplier tube (spectral range $150-650 \mathrm{~nm}$, transit time jitter $40 \mathrm{ps}$ fwhm) or a quartz windowed $\mathrm{GaAs}(\mathrm{Cs})$ photomultiplier tube (spectral range $160-930 \mathrm{~nm}$, transit time jitter $4 \mathrm{~ns}$ fwhm). Decay time spectra are acquired using a TDC having $40 \mathrm{ps}$ fwhm resolution over a $84 \mathrm{~ms}$ dynamic range. A computer controlled monochromator can be inserted into the optical path to measure the emission spectrum or wavelength resolved decay time spectrum. A computer controlled sample changer allows up to 64 samples to be measured without intervention.
\end{abstract}

\section{INTRODUCTION AND MOTIVATION}

A Pulsed X-ray Facility has been developed at Lawrence Berkeley Laboratory (LBL) to aid an ongoing search for new scintillators for gamma ray detection. While virtually all applications that utilize scintillators for gamma ray detection would benefit from improved scintillators, almost every application has different requirements, and so the scintillation properties of currently used scintillators vary significantly. These properties include decay time (sub-nanosecond to hundreds of milliseconds), luminous efficiency (which varies over two orders of magnitude) spectral range (from the infrared to the vacuum ultraviolet), and physical form (from transparent crystals up to a meter in size to micron sized powders).

The Facility accurately measures scintillator decay time and emission spectra, as well as estimates luminous efficiency, over a wide range of times, wavelengths, and signal intensities. The design priorities included compact size, low cost, and the ability to measure multiple samples under computer control. The critical component is a light-excited $x$-ray tube that produces brief $(\sim 100 \mathrm{ps})$ pulses of $x$-rays [1]. The design and performance of the pulsed $x$-ray source are described in $[2$, 3]; the primary purpose of this paper is to describe and characterize the remainder of the data collection system.

\section{GENERAL SYSTEM DESIGN}

The LBL Pulsed X-ray Facility is shown diagramatically in figure 1 . X-rays produced by the pulsed $x$-ray tube impinge upon the sample, and the fluorescent emanations (if any) are focused by a lens system and converted into electrical pulses by a photomultiplier tube (PMT). A monochromator system

\footnotetext{
* This work was supported in part by the U.S. Department of Energy contract DE-AC03-76SF00098 and in part by Public Health Service Grants Nos. P01 25840 and R01 CA48002.
}

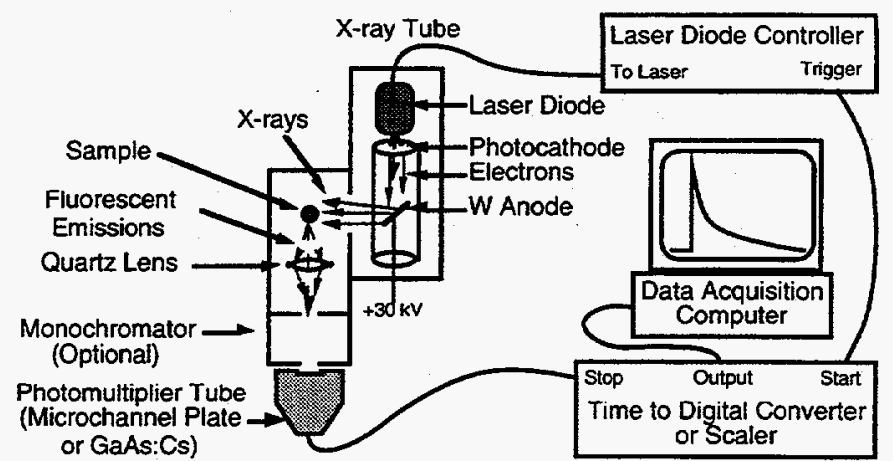

Figure 1. Schematic of the LBL Pulsed X-ray Facility. Light from a laser diode excites the photocathode, liberating photoelectrons that are accelerated to the tungsten anode, creating $x$-rays on impact. The $x$-rays excite the sample and the resulting fluorescent emissions are detected with a photomultiplier tube and recorded.

can be inserted into the optical path to provide wavelength selectivity. A variety of conventional nuclear electronics is used to process the data, including a time to digital converter (TDC) to record the arrival time of the optical signal and a scaler to count the number of pulses observed. All facets of the Facility are under the control of a personal computer.

Figure 2 is a photograph of the pulsed $x$-ray source. The steel box on the table top is the general purpose sample chamber, and directly behind it (hidden from view) is the $x$-ray tube. The laser diode is mounted above the $x$-ray tube, and the laser diode controller is on the stand above the table top. The sample chamber is evacuated by the pump under the table top. Scintillation light passes through the quartz telescope on the right side of the sample chamber to a microchannel plate PMT. The $x$-ray tube high-voltage power supply and the control panel, which includes extensive safety interlocks and a meter for monitoring the $\mathrm{x}$-ray tube cathode current, are mounted in the lower right portion of the table.

\section{INDIVIDUAL COMPONENTS}

\section{A. X-ray Source}

The main components of the pulsed $x$-ray source are the light-excited $\mathrm{x}$-ray tube and the laser diode shown in Figure 1. The $x$-ray tube is a Hamamatsu N5084 Light-Excited X-ray Tube, which is essentially a single-stage photomultiplier tube with an S-20 photocathode that releases electrons when light is absorbed. The electrons are accelerated across $30 \mathrm{kV}$ into a tungsten anode, where bremsstrahlung $\mathrm{x}$-rays are produced upon impact. The light-excited design of the $\mathrm{x}$-ray tube makes it possible to generate $x$-ray pulses by directing short pulses of light onto the photocathode. The time structure of the $x$-rays is that of the incident light convolved with the broadening due to the $\mathrm{x}$-ray tube, estimated to be $31 \mathrm{ps}$ fwhm.

Light pulses are generated by a Hamamatsu PLP-01 Light Pulser with a LDH065 laser diode head and C3551-01 


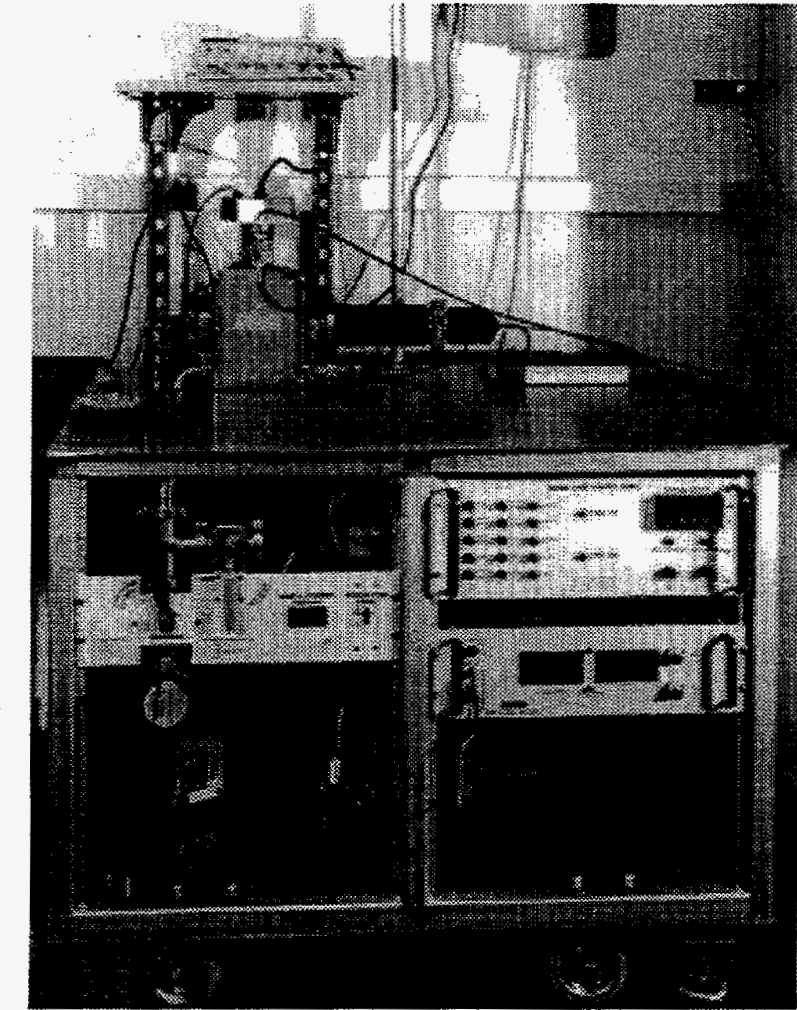

Figure 2. Photograph of the LBL Pulsed X-ray Facility.

controller that produces fixed width pulses ( $97 \mathrm{ps}$ fwhm) of $650 \mathrm{~nm}$ light at a repetition rate that is adjustable from 0 to $10 \mathrm{MHz}$. The peak power is $100 \mathrm{~mW}$, corresponding to $\sim 10^{7}$ photons per pulse. A Peltier junction maintains the temperature of the laser diode at $26^{\circ} \pm 1^{\circ} \mathrm{C}$ for pulse timing stability.

The $\mathrm{x}$-ray tube emits approximately 200 bremsstrahlung $\mathrm{x}$ rays per steradian (mean energy $11.1 \mathrm{keV}$ ) per pulse. As most of the samples are in $1 \mathrm{~mm}$ wall thickness quartz cuvettes, a large fraction of the lower energy $x$-rays are absorbed in the cuvette, but do not contribute to the fluorescence signal from the sample and generate background fluorescence from the quartz. Therefore, a $0.51 \mathrm{~mm}$ aluminum filter is placed in the $\mathrm{x}$-ray beam to absorb low-energy $\mathrm{x}$-rays. After filtration the flux is reduced to $40 \mathrm{x}$-rays per steradian with a mean $\mathrm{x}$-ray energy of $18.5 \mathrm{keV}$. A background $\mathrm{x}$-ray flux is generated in the absence of laser diode light by photocathode dark current in the $\mathrm{x}$-ray tube. However, this flux is quite small; the ratio of the "background" flux to the "laser on" flux is $1.5 \times 10^{-5}$.

As configured above, the $x$-ray tube operates at $1 / 500$ of its maximum average current of $50 \mu \mathrm{A}$. We plan to increase the number of x-rays per pulse (at the expense of a wider pulse) by using a $630 \mathrm{~nm}$ laser diode with $15 \mathrm{~mW}$ peak power. The laser diode controller will have adjustable pulse widths $\geq 1 \mathrm{~ns}$ fwhm. A 1 ns pulse will produce $50 \%$ more $x$-rays than a pulse from the present laser diode, and the pulse width can be lengthened arbitrarily, allowing operation at the maximum $x-$ ray tube current when pulsed with $33 \%$ duty factor.

\section{B. Sample Holder}

Either of two sample holders can be attached to the $x$-ray source. Both are constructed from $6 \mathrm{~mm}$ thick stainless steel, locate the sample approximately $50 \mathrm{~mm}$ from the tungsten anode, and have an evacuated ( $<50$ millitorr) sample chamber. The evacuation serves two purposes - to eliminate the background caused by air scintillation and to allow a single vacuum switch to be used as an $\mathrm{x}$-ray safety interlock for the multiple access ports in the sample holders.

One sample holder is a general purpose, single sample holder. Its main component is a brass shelf with fiducial marks that allow arbitrarily shaped samples to be positioned in the $\mathrm{x}$-ray beam at the focus of the optical detection system. However, most samples are in powdered form inside $5 \mathrm{~mm}$ outside diameter, $3 \mathrm{~mm}$ inner diameter, $50 \mathrm{~mm}$ long quartz cuvettes (a short version of a standard "NMR tube"), so this holder also includes a fixture for holding such cuvettes. The brass shelf has internal tubes for flowing cooling fluid, an internal resistive heater, and a thermocouple attached to a temperature controller, and so can be held at a fixed temperature ranging from $-200^{\circ} \mathrm{C}$ to $+200^{\circ} \mathrm{C}$.

The other sample holder holds up to 64 samples in cuvettes. The samples are stored out of the $x$-ray beam in a rotating carousel, then individually lowered into the $x$-ray beam / optical focus. Both the carousel rotation and sample lowering / raising are computer controlled through stepping motors, and the current from a silicon solid state $x$-ray detector is also monitored by the computer to ensure that the sample drops to the proper position and occludes the $x$-ray beam. This sample holder is only capable of room temperature operation.

\section{Optical Path}

The samples are positioned at the focus of an optical system whose first element is a $50 \mathrm{~mm}$ diameter, $50 \mathrm{~mm}$ focal length quartz lens. The optical axis of this system is perpendicular to the $x$-ray beam. A variety of optical components can also be inserted into the optical path, including quartz focusing lenses, computer selectable cutoff filters with transmission edges ranging from the ultraviolet through the visible, and a computer controlled $0.125 \mathrm{~m}$ monochromator (1200 line/mm gratings blazed for $300 \mathrm{~nm}$ and $500 \mathrm{~nm}$ ). All optical components are coupled using quick connect vacuum flanges to enable light tight, mechanically rigid, rapidly changeable connections.

\section{Optical Detection}

A variety of photomultiplier tubes are available for converting the fluorescence photons into electrical pulses. These include a sapphire windowed microchannel plate PMT (41 ps fwhm transit time jitter or $\mathrm{ttj}, 150-650 \mathrm{~nm}$ spectral range), a quartz windowed GaAs(Cs) PMT (4 ns fwhm ttj, 160$930 \mathrm{~nm}$ spectral range), and several quartz windowed bialkalai PMTs of conventional design ( $0.5 \mathrm{~ns}$ fwhm ttj, 160-650 nm spectral range). All PMTs can be housed in thermoelectric coolers capable of cooling to $-20^{\circ} \mathrm{C}$ to reduce dark current.

\section{Electronics}

The PMT output is amplified ( $85 \mathrm{~dB}$ gain, $3 \mathrm{GHz}$ bandwidth) and converted into a logic pulse with good timing 
Presented at LUMDETR '94 (Tallinn, Estonia), submitted to Radiation Measurements

accuracy via a Ortec 9307 pico-timing discriminator. The logic pulses are counted with a $300 \mathrm{MHz}$ scaler and the time difference between the laser diode trigger pulse (which is synchronized to the diode laser light output within $10 \mathrm{ps}$ ) and this logic pulse digitized with a Highland M-690 TDC that digitizes in 20 ps bins (albeit 40 ps fwhm resolution) over an $84 \mathrm{~ms}$ dynamic range.

Several custom circuits enhance the data collection rate and data. An interface to a pair of 14 address bit histogramming memory modules allows readout and histogramming of the TDC to occur in $1 \mu \mathrm{s}$. The data is histogrammed unaltered in one of the memory modules, allowing high accuracy (determined by the instrumental response) at times near the excitation time, and is shifted by a computer selected number of bits before histogramming in the second memory module to cover a larger dynamic range, but with less accuracy (determined by the number of bits shifted). Another circuit detects events with more than one fluorescent photon per excitation pulse, thus increasing the data collection rate and accuracy as described in [4]. This circuit interacts with the histogramming memory interface circuit to inhibit storage of events with multiple fluorescent photons.

\section{E. Computer}

All data acquisition and control is done with a personal computer interfaced to CAMAC electronics. Where possible, data accumulation is done in dedicated hardware to maximize accumulation rate.

\section{MEASUREMENTS}

\section{A. Decay Time Spectra}

Decay time spectra are accumulated using the delayed coincidence method [5] with the modifications described in [4]. An upper limit on the system impulse response time, which includes the laser diode, $x$-ray tube, microchannel plate PMT, amplifier, timing discriminator, and TDC, was measured to be $120 \mathrm{ps}$ fwhm. This upper limit was obtained by exciting malachite green oxalate crystals and recording the resulting $(120 \mathrm{ps})$ width of the fluorescent decay time spectrum. Malachite green oxalate is used as a fast laser dye, and while its $x$-ray excited decay time in crystal form is not known, its optically excited fluorescent decay time is $\ll 100 \mathrm{ps}$ in a large variety of solvents and concentrations [6].

Decay time data are rebinned into bins with exponentially increasing width, so that the bin width divided by time from excitation is a constant $1 \%$. This greatly reduces the storage space required - a spectrum with 20 ps bins covering a $12 \mu$ s dynamic range would require over 600,000 bins in uncompressed form but only 1,300 bins after compression. This also has a major impact on the computation time necessary to fit the decay time spectrum, as the observed value and the fit value must be compared bin by bin on each iteration of the fitting procedure.

Figure 3 shows the decay time spectrum obtained for a 1000 second data collection of a crystalline sample of $\mathrm{CsI}_{0.9} \mathrm{Br}_{0.1}$. This data set shows the value of obtaining spectra with both high resolution and large dynamic range, as it has structure on several different time scales. At short times (figure 3a) there is a fast, $1.5 \mathrm{~ns}$ decay. At intermediate times (figure $3 \mathrm{~b}$ ) there is a rise time followed by a $565 \mathrm{~ns}$ decay time, while at long decay times (figure $3 \mathrm{c}$ ) there is a $152 \mu \mathrm{s}$ apparent decay time that is due to the bias caused by events with more than one fluorescent photon per excitation pulse (multiple photon rejection was not performed for this data set). In general, data collection times are reasonably short in this data taking mode. For a powdered sample of BGO scintillator, over 5000 events per second are accumulated.

\section{B. Emission Spectra}

Emission spectra are acquired by inserting the monochromator into the optical path and measuring the count rate in the PMT as a function of monochromator wavelength. For compounds with a complex spectra, such as that from $\mathrm{ScPO}_{4}: 2 \% \mathrm{Nd}$ shown in figure 4 , separate data collections with each of the two monochromator gratings ( 300 and $500 \mathrm{~nm}$ blaze) are necessary. Cutoff filters can be inserted into the optical path to reduce the passage of higher order light through the monochromator. The relative quantum efficiency for the combination of the monochromator and one of the conventional PMTs has been measured, allowing the data to be corrected for the spectral dependence of the detector system. Because of the small spectral width passed by the monochromator ( $12 \mathrm{~nm}$ fwhm typically), data collection rates are low. For a powdered sample of BGO scintillator, the counting rate at the emission spectrum peak is approximately 5 photons per second with the current laser diode. Once the
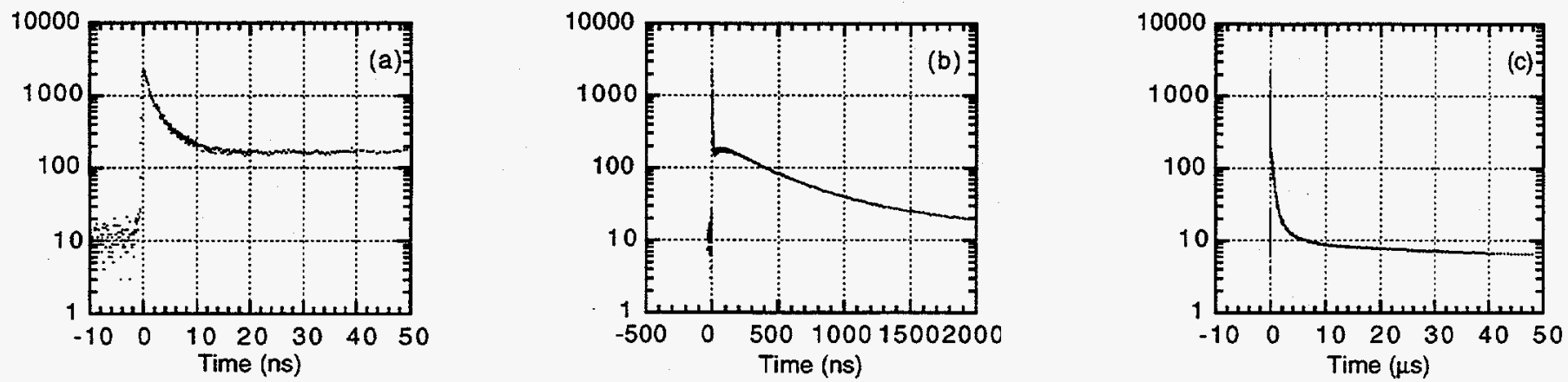

Figure 3. Fluorescent decay time spectrum from $\mathrm{CsI}_{0.9} \mathrm{Br}_{0.1}$. The data were collected as a single data set, but are displayed in short (a), medium (b), and long (c) time scales to demonstrate the different structure visible at different time scales. 
Presented at LUMDETR '94 (Tallinn, Estonia), submitted to Radiation Measurements

LBL-36271

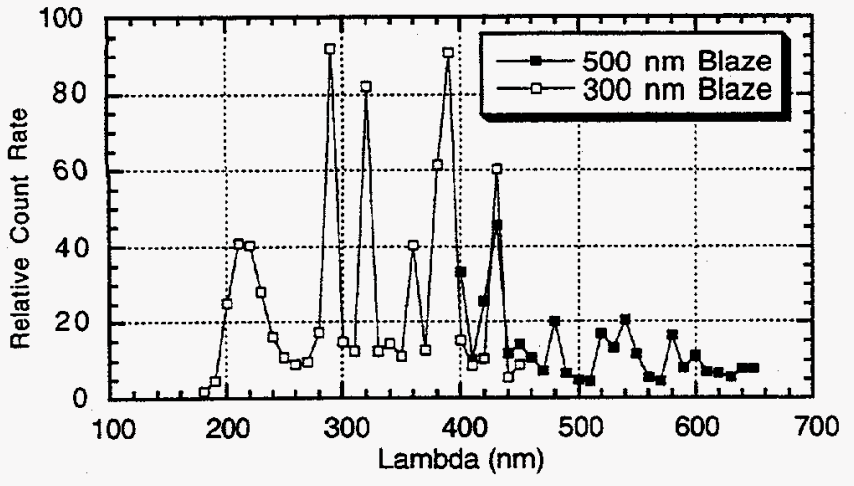

Figure 4. Emission spectrum of $\mathrm{ScPO}_{4}: 2 \% \mathrm{Nd}$.

new laser diode is installed, it is expected to increase to 2500 photons per second.

\section{Wavelength Resolved Decay Time}

Wavelength resolved decay time spectra can be accumulated by inserting the monochromator (and order sorting filters if desired) into the optical path and measuring the decay time. The data accumulation rate for such measurements is the same as for emission spectra but three orders of magnitude lower than for decay time spectra due to the limited amount of light passed by the monochromator.

\section{Scintillation Efficiency}

The scintillation efficiency can be estimated by comparing the counting rate observed in an unknown sample to that from a sample of known scintillation efficiency. This estimate has significant uncertainty, as the observed counting rate is depends strongly on the (unknown) optical attenuation length and the x-ray attenuation length of the samples as well as their scintillation efficiency. However, if the optical attenuation length is assumed to be the same for all samples, the $\mathrm{x}$-ray attenuation length can be corrected for using the method described in [7] and an estimate of the scintillation efficiency obtained. These estimates can be surprisingly accurate - the estimates using $x$-ray excitation of powdered samples for a number of previously unmeasured scintillators [8] are within $10 \%$ of later measurements with good optical quality crystals.

\section{E. Afterglow}

Scintillation decay times longer than $50 \mu$ s are difficult to measure using the conventional delayed coincidence method, as the pulse repetition rate and scintillation collection efficiency are necessarily low. However, we have previously described a method using a multi-hit TDC that would allow such decay times to be measured with relative ease [4]. This pulsed $x$-ray source, with the more powerful laser and a multi-hit TDC, has the capability to make these measurements quickly and accurately, and so provide measurements of scintillator afterglow.

\section{CONCLUSIONS}

The LBL Pulsed X-ray Facility is a compact, table-top device capable of characterizing scintillators with a wide range of decay times, emission spectra, luminous efficiency, and physical forms. Its time resolution is less than $120 \mathrm{ps}$ fwhm and with a well characterized impulse response it can be used to determine fluorescence decay times to within $50 \mathrm{ps}$. The width and repetition rate of the $\mathrm{x}$-ray pulses can easily be varied by adjusting the laser diode pulse characteristics. The spectral range of the detection system is from $150 \mathrm{~nm}$ to $950 \mathrm{~nm}$, so a monochromator can readily be incorporated to measure scintillation emission spectra or to obtain wavelength resolved decay time spectra. Surprisingly accurate estimates of luminous efficiency can be made, and the inclusion of a TDC with multi-hit capability should allow this device to extend its accurate decay time measurement range from $50 \mu \mathrm{s}$ to $50 \mathrm{~ms}$, which is useful for characterizing scintillator afterglow.

\section{ACKNOWLEDGMENTS}

This work was supported in part by the Director, Office of Energy Research, Office of Health and Environmental Research, Medical Applications and Biophysical Research Division of the U.S. Department of Energy under contract No. DE-AC03-76SF00098, and in part by Public Health Service Grant Nos. P01 HL25840, and R01 CA48002 awarded by the National Heart Lung and Blood and National Cancer Institutes, Department of Health and Human Services. Reference to a company or product name does not imply approval or recommendation by the University of California or the U.S. Dept. of Energy to the exclusion of others that may be suitable.

\section{REFERENCES}

[1] Oba K. Instrument to measure fluorescence which has occurred in a sample stimulated by $x$-rays, U.S. patent $\# 4,724,536$, issued to Hamamatsu Photonics, Japan, 1988.

[2] Derenzo SE, Moses WW, Blankespoor SC, et al. Design of a pulsed $\mathrm{x}$-ray system for fluorescent lifetime measurements with a timing resolution of 109 ps. IEEE Trans. Nucl. Sci. NS-41: pp. 629-631, 1994.

[3] Blankespoor SC, Derenzo SE, Moses WW, et al. Characterization of a pulsed x-ray source for fluorescent lifetime measurements. IEEE Trans. Nucl. Sci. NS-41: pp. 698-702, 1994.

[4] Moses WW. A method to increase scintillation lifetime measurement rates using a multi-hit TDC. Nucl. Instr. Meth. A-336: pp. 253-261, 1993.

[5] Bollinger LM and Thomas GE. Measurement of the time dependence of scintillation intensity by a delayedcoincidence method. Rev Sci lnstr 32: pp. 1044-1050, 1961.

[6] Yu W, Pellegrino $F$, Grant $M$, et al. Subnanosecond fluorescence quenching of dye molecules in solution. $J$. Chem. Phys. 67: pp. 1766-1773, 1977.

[7] Derenzo SE, Moses WW, Cahoon J, et al. Prospects for new inorganic scintillators. IEEE Trans. Nucl. Sci. NS-37: pp. 203-208, 1990.

[8] Derenzo SE, Moses WW, Cahoon JL, et al. X-ray fluorescence measurements of 412 inorganic compounds. Proceedings of The IEEE Nuclear Science Symposium, pp. 143-147, (Edited by G. T. Baldwin), Santa Fe, NM, 1991. 